\title{
Online SOM-based level set model for image segmentation
}

\author{
Xiaomin Xie \\ College of Mechanical \& Electrical Engineering, Hohai University, Changzhou, 213022, China \\ yu_jian_wo@126.com
}

Keywords: SOM, Level set model; Local information; Image segmentation.

\begin{abstract}
In this paper, a novel region based active model integrating self organizing map (SOM) for image segmentation is proposed. The SOM is utilized to describe image intensity distributions in an unsupervised way and establish the entire weights set of the neurons. Then, the weights are divided into two branch sets depending on whether the weight is larger than the weight mean. Further we obtain the means of two weights subsets, which could be regarded as the average intensities of the background and foreground, respectively. In addition, the global intensities updated during contour evolution are replaced by the prototypes of the entire weights set according to certain rule. Along with the two weight means, the global means are averaged to create the threshold for the whole image to guide the contour movement. The proposed model could be successfully applied for image segmentation. Experiment results demonstrate the effectiveness of our model.
\end{abstract}

\section{Introduction}

Image segmentation has long been thought to be vital for machine learning. Active contour model (ACM) [1-2], which devotes to guiding the contour to move towards the true object boundaries, has been paid more and more attention in image segmentation. It works mainly based on the minimum of the energy function which is constructed by the image statistical information. CV model [1], one of the most famous region-based ACMs, assumes that the image intensities obey Gaussian intensity distributions, and hence approximates the image by two piecewise-constants. Similarly, some of the ACMs employ certain probability model to approximate the intensity distributions [1-2]. And the prior knowledge models are usually applied to simulation environment with great success. However, in certain cases, these models, which are strongly dependent on the statistical assumptions about the image intensity distributions, would limit their practical applications, e.g., in dealing with images that contain noise and weak edges. The model misspecification, in general, is responsible for the dissatisfactory segmentation results.

To solve the problem, the CSOM-CV [3-4] combines the advantage of the SOM and ACM and trains two SOMs concurrently: one for the foreground and the other for the background. They replace the region mean intensities by the related prototypes coming from the two trained networks. The SOMs possess the ability of predicting the image intensity distribution using the topology preservation property. Hence, some hybrid ACM models in combination with the SOMs are proposed in the literature to avoid the negative effect of model misspecification. However, there are still some problems, for instance, the foreground and background regions for training are settled in advance. Hence, [5] proposes an unsupervised SOM-based CV model i.e. SOMCV which trains a set of neurons model globally by a self-organization learning procedure.

Inspired by the SOMCV and online region-based active contour model i.e. ORACM [6], a novel active model for image segmentation is presented in this paper. The SOM is utilized to describe image intensity distributions in an unsupervised way and establish the entire weights set of the neurons. Then, the weights are divided into two branch sets depending on whether the weight is larger than the weight mean, and thus we obtain the means of two weights subsets which could be regarded as the average intensities of the background and foreground, respectively. In addition, the global intensities updated during contour evolution are replaced by the prototypes of the entire weights set according to certain rule. Along with the two weight means, global means are averaged to create the threshold for the whole image to guide the contour towards the desired boundaries. 


\section{Related Works}

CV Model. Chan and Vese propose an active contour model (i.e. CV model [1]), which energy in term of level set function is expressed as:

$$
E\left(\phi(x), m_{1}, m_{2}\right)=\int\left(I-m_{1}\right)^{2} H(\phi(x)) d x+\int\left(I-m_{2}\right)^{2}(1-H(\phi(x))) d x+\mu \int|\nabla H(\phi)| d x
$$

Where: $I$ be a given image, and $\Omega \subset \mathrm{R}^{n}$ be the image domain, $H(\phi)$ is the Heaviside function which specifies the interior of zero level set function, and vice versa. $m_{1}$ and $m_{2}$ designate two constants that approximate the image intensities in interior and exterior of zero level set function, respectively. The third length term with the scaling parameter ${ }^{\mu}$ is responsible for the regularization of contour. Minimizing the energy in (1) through the steepest descent algorithm, we obtain:

$$
m_{1}=\frac{\int I(x) H(\phi(x)) d x}{\int H(\phi(x)) d x}, m_{2}=\frac{\int I(x)(1-H(\phi(x))) d x}{\int(1-H(\phi(x))) d x}
$$

ORACM model. ORACM model [6], which performs a sort of block threshold process in each iteration, uses a user defined active contour at the initialization step and then continuously updates it. Its efficient level set updating formulation is given as follows:

$$
\frac{\partial \phi}{\partial t}=H(\operatorname{spf}(I(x))) \cdot \phi(t)
$$

Where $\operatorname{spf}($.$) is the signed pressure function [6].$

\section{The Proposed Model}

The proposed model starts by training one SOM for the whole image globally. As for training session, the proposed model shares the same configurations as the SOM used in the SOMCV (Turn to [5] for more details). After the training, a set of prototypes $\mathbf{W}$ which describes the global intensity distribution of the image, is obtained to be the primary training set.

Different from SOMCV, we further average the prototypes set to obtain its mean $\bar{w}$. Then, the set $\mathbf{W}$ is divided into two subsets $\mathbf{W}_{s}$ and $\mathbf{W}_{l}$ taking the $\bar{w}$ as the threshold. Then the means of the two subsets are obtained, which are:

$$
\begin{aligned}
& \left\{\begin{array}{l}
\mathbf{W}_{s}=\{w \leq \bar{w} \mid w \in \mathbf{W}\} \\
\mathbf{W}_{l}=\{w \geq \bar{w} \mid w \in \mathbf{W}\}
\end{array}\right. \\
& \left\{\begin{array}{l}
\bar{w}_{s}=\operatorname{mean}\left(\mathbf{W}_{s}\right) \\
\bar{w}_{l}=\operatorname{mean}\left(\mathbf{W}_{l}\right)
\end{array}\right.
\end{aligned}
$$

Further, the global intensities means $m_{1}$ and $m_{2}$ are taken as the input of the prototypes $\mathbf{W}$ to find their closest prototypes:

$$
w_{m i}:=\arg \min \left|\mathbf{W}-m_{i}(x)\right| \quad i=1,2
$$

Then the block threshold could be calculated as:

$$
T h=\operatorname{mean}\left(\bar{w}_{s}, \bar{w}_{s}, w_{m 1}, w_{m 2}\right)
$$

Thus, the signed pressure function $\operatorname{spf}($.$) could be defined as:$

$$
\text { spf }=(I(x)-T h) / \max (I(x)-T h)
$$

Then we put the expression into (3) to obtain the contour evolution. When the iterative stopping condition is satisfied, the contour is exactly on the boundary of the object. And then the Gaussian filter is used to smooth the level set. 


\section{Experiments and analysis}

In this section, numerical examples are shown to validate the effectiveness of the proposed model. We set the default scale parameter $\sigma=1$ for the Gaussian filter.

(a)
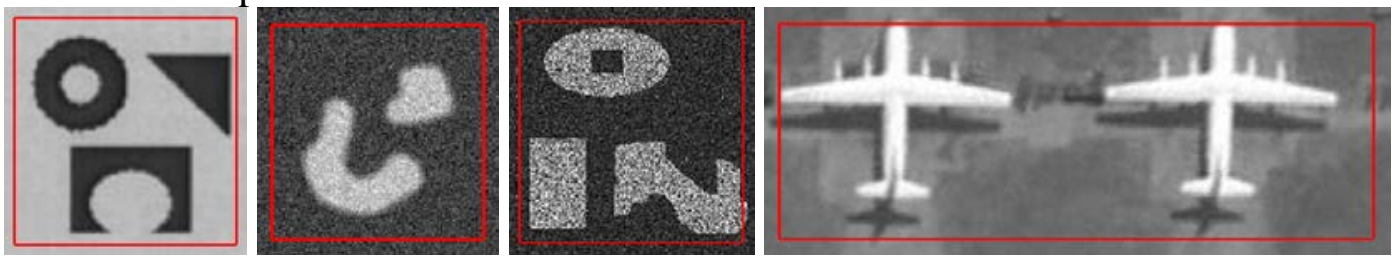

(b)
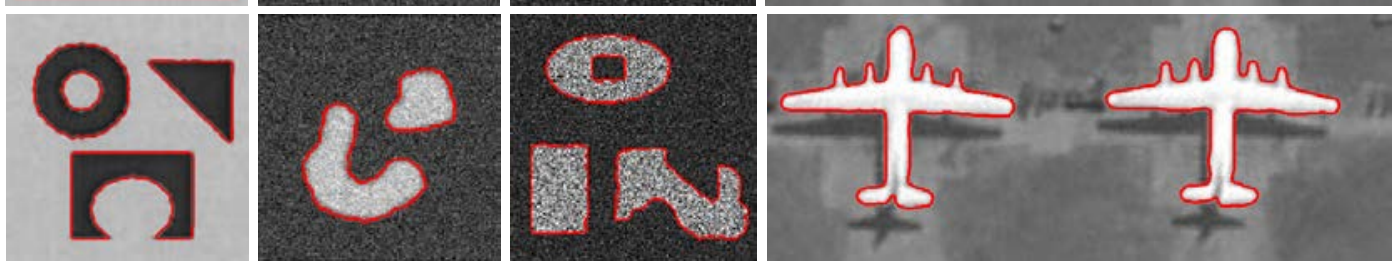

Fig. 1 Segmentation results of our model on a set of images: (a) Initial contour, (b) Final contour of our model

Experiments in Fig.1 are conducted on a set of images to demonstrate the segmentation performance. There are four images to be test: the first one is a synthetic image which has three objects with different shapes, the second synthetic image is corrupted by Gaussian noise, the third synthetic image is added with Speckle noise, and the last one is a real plane image. The initial contour for each image is shown in Fig.1 (a). As can be seen from Fig.1 (b), using the proposed model, we could obtain satisfied segmentation results after several iterations.

The proposed model adopts the online region-based ACM model, and hence has the advantages over the SOMCV [5] model in terms of speed. We conduct the experiment to compare the MOSCV and the proposed model, where the test images have been shown in Fig.1. Benefited from the same principle, the two models share the similar segmentation results. However, their elapsed times are different, which are shown in Table 1. As can be seen from Table 1, the proposed model takes less time.

Table 1 The elapsed time of SOMCV model [5] and our model in Fig.1

\begin{tabular}{ccc}
\hline No. & SOMCV & Ours \\
\hline 1 & 3.4931 & 2.9224 \\
2 & 3.4984 & 2.7843 \\
3 & 3.5131 & 2.8757 \\
4 & 3.5091 & 2.9557 \\
\hline
\end{tabular}

\section{Summary}

In this paper, we propose a novel region based active contour model. The SOM is adopted to obtain a set of of prototypes and further two subsets. The means of the two subsets, as well as the global intensities means updated by the contour are used to estimate the block threshold for the online region-based ACM which is a framework employed in this paper. Experiments on synthetic and real images demonstrate the effectiveness of the proposed model.

\section{Acknowledgements}

This work was supported by * the Fundamental Research Funds for the Central Universities * (grant B2X/16B101-02).

\section{References}

[1] T.F. Chan, L.A. Vese, Active Contours Without Edges, IEEE Trans. Image Process. 10 (2) (2001) 266-277. 
[2] S.G. Liu, Y.L. Peng, A local region-based Chan-Vese model for image segmentation, Pattern Recogn. 45 (2012) 2769-2779.

[3] M.M. Abdelsamea, G. Gnecco, M.M. Gaber, An efficient self organizing active contour model for image segmentation, Neurocomputing 149 (2015) 820-835.

[4] M.M. Abdelsamea, G. Giorgio, M.M. Gaber, A concurrent SOM-Based Chan-Vese model for image segmentation, Proc. WSOM. 295 (2014) 199-208.

[5] M.M. Abdelsamea, G. Gnecco, M.M. Gaber, A SOM-based Chan-Vese model for unsupervised image segmentation, Soft Comput. (2015) 1-21.

[6] M.F. Talu, ORACM: Online region-based active contour model, Expert Syst. Appl. 40 (2013) 6233-6240. 